\title{
HPV Catch-up Vaccination is Effective but Cervical Screening Should Continue
}

Nick F. Hallam*

Colposcopy Clinic, Women's Outpatients, Cumberland Infirmary, Carlisle, England, United Kingdom

\section{Article Info}

\section{Article Notes}

Received: June 02, 2021

Accepted: June 22, 2021

\section{*Correspondence:}

${ }^{*}$ Dr. Nick F. Hallam, Colposcopy Clinic, Women's Outpatients, Cumberland Infirmary, Carlisle, England, United Kingdom; Email: Nick.Hallam@uwclub.net.

C $2021 \mathrm{Hallam}$ NF. This article is distributed under the terms of the Creative Commons Attribution 4.0 International License.

\section{Keywords}

HPV catch-up bivalent vaccination

Efficacy

Cervical screening

\section{ABSTRACT}

This short communication reports additional research that extends the previously published article - Commentary: HPV Catch-Up Vaccination Reduces the Prevalence of HPV 16 and 18 Infections and Cervical Disease: A Retrospective Study. ${ }^{1}$ One limitation of that study was uncertainty as to whether the catch-up cohort had actually received HPV (human papillomavirus) vaccination. That information has now been obtained. 87 (59\%) of the 147 patients in the catch-up cohort had received at least one dose of HPV bivalent vaccine. 69 of these (representing $79 \%$ of those vaccinated) had received three doses (as recommended at the time). Both the vaccinated and unvaccinated subsets of the catch-up cohort show a significant reduction in the prevalence of HPV 16 and/or 18 (with/without other high-risk types 31, 33, 35, 39, 45, 51, 52, 56, $58,59,66$ and 68 ) and of high grade cervical disease compared to an earlier unvaccinated cohort. These results confirm the efficacy of HPV catch-up vaccination and the existence of herd immunity following the introduction of national HPV vaccination campaigns. However, 34 patients $(23 \%)$ in the catchup cohort had high grade disease (cervical intraepithelial neoplasia [CIN] 2 or worse), 16 of whom had been vaccinated (12 with three doses, one with two doses and three with one dose of HPV bivalent vaccine) and four of those vaccinated had HPV 16 and/or 18 (with/without other high-risk types), the rest had other HPV high risk types. This emphasises the importance of maintaining cervical screening alongside HPV vaccination.

\section{Background}

The initial study was carried out in North Cumbria, England, United Kingdom (UK). The patients involved were two cohorts of women attending for colposcopy after their first HPV primary cervical screening test at 25 years of age. The test used detected HPV high-risk types 16, 18, 31, 33, 35, 39, 45, 51, 52, 56, 58, 59, 66 and 68. One cohort (tested in 2014-2015) had not been eligible for HPV bivalent (types 16 and 18) vaccination which was introduced in 2008, the other (tested in 2018-2019) was eligible for the HPV bivalent vaccination catch-up campaign for those who were 14-18 years old in 2008. Outcome measures were the HPV primary cervical screening test results and histology results at first colposcopy visit. We found a reduction in the prevalence both of HPV 16 and/ or 18 (with/without other high-risk types) from 53\% (103 of 193 patients) to $16 \%$ (23 of 147 patients; $95 \% \mathrm{CI}=27.2 \%-45.6 \%$, Chisquared $=48.9, \mathrm{p}<0.0001)$ and of high grade cervical disease (CIN 2 or worse) from $54 \%$ (104 of 193 patients) to 23\% (34 of 147 patients; $95 \% \mathrm{CI}=20.8 \%-40.2 \%$, Chi-squared $=33.1, \mathrm{p}<0.0001$ ) in the bivalent HPV vaccine catch-up 2018-2019 cohort compared with the unvaccinated 2014-2015 cohort.

One limitation of this study, which was mentioned in the 
earlier commentary ${ }^{1}$ and reflects the lack of a centralised integrated data base combining vaccination and screening records in England, was that we described the catch-up cohort as being 'eligible' for HPV vaccination rather than being able to state whether or not they actually received it. After contacting the patients' community medical practitioners, who hold the relevant records, the HPV vaccination status of those in the catch-up cohort was obtained.

\section{New results}

87 (59\%) of the 147 patients in the catch-up cohort had received at least one dose of HPV bivalent vaccine. $83(94 \%)$ of these were vaccinated at 15-16 years old, two at 18 years old and one each at 14 and 17 years old respectively. 69 (79\%) of those vaccinated received three doses of vaccine (the recommendation at that time), 9 received two doses and 9 received one dose. There is evidence ${ }^{2-4}$ that even a single dose of HPV vaccine may confer significant protection and therefore all 87 patients have been included in the vaccinated subset of this cohort for further statistical analysis. The remaining 60 (41\%) patients in the catch-up cohort had no record of HPV vaccination and form the unvaccinated subset.

The outcome measures were, as previously, the HPV primary cervical screening test results and histology results at first colposcopy visit. Comparing the two subsets in the catch-up cohort with each other there was a significant reduction in the prevalence of HPV 16 and/ or 18 (with/without other high-risk types) from $25 \%$ (15 of 60 patients) to $8 \%$ (7 of 87 patients; $95 \% \mathrm{CI}=5.0 \%$ 29.9, Chi-squared $=8.0, p=0.0046$ ) in the vaccinated subset compared to the unvaccinated one, but there was a non-significant reduction in high grade cervical disease (CIN 2 or worse) from 30\% (18 of 60 patients) to $18.3 \%$ (16 of 87 patients; $95 \% \mathrm{CI}=-2.1 \%-25.9 \%$, Chi-squared $=$ $2.7, \mathrm{p}=0.0991$ ) in the vaccinated subset compared to the unvaccinated one.

Comparing each subset separately with the original unvaccinated 2014-2015 cohort showed significant reductions in both parameters (the prevalence of HPV 16 and/or 18 [with/without other high-risk types] and high grade cervical disease [CIN 2 or worse]) for both subsets. For the vaccinated subset these reductions were a) from $53.4 \%$ (103 of 193 patients) to $8 \%$ (7 of 87 patients; $95 \%$ $\mathrm{CI}=35.0 \%-53.4 \%$, Chi-squared $=51.6, \mathrm{p}<0.0001)$ for the prevalence of HPV 16 and/or 18 (with/without other high-risk types) and b) from 53.9\% (104 of 193 patients) to $18.3 \%$ ( 16 of 87 patients; $95 \% \mathrm{CI}=23.8 \%-45.2 \%$, Chi-squared $=30.9, \mathrm{p}<0.0001$ ) for high grade cervical disease (CIN 2 or worse). The equivalent reductions for the unvaccinated subset were a) from 53.4\% (103 of 193 patients) to $25 \%$ (15 of 60 patients; $95 \% \mathrm{CI}=14.3 \%$ -
$39.9 \%$, Chi-squared $=14.8, \mathrm{p}=0.0001)$ and $\mathrm{b}$ ) from $53.9 \%$ (104 of 193 patients) to $30 \%$ (18 of 60 patients; $95 \% \mathrm{CI}=$ 9.5\%-36.1\%, Chi-squared $=10.4, p=0.0012$ ).

In our study a total of 34 patients (23\%) in the catch-up cohort had high grade disease (CIN 2 or worse). We now know that 16 of these had been vaccinated (12 with three doses, one with two doses and three with one dose of HPV bivalent vaccine) and that four of those vaccinated had HPV 16 and/or 18 (with/without other high-risk types), the rest had other HPV high-risk types. The remaining 18 patients of the 34 were unvaccinated, 9 of these had HPV 16 and/or 18 (with/without other high-risk types), the rest had other HPV high-risk types.

\section{Discussion}

These results confirm the efficacy of HPV catch-up vaccination and the existence of herd immunity following the introduction of national HPV vaccination campaigns. ${ }^{5-9}$ But neither vaccination (even with the more recent Gardasil 9-valent HPV vaccine ${ }^{10}$ ) nor herd immunity currently protect against all high-risk HPV types or the associated high grade cervical disease.

This emphasises the need to maintain cervical screening alongside HPV vaccination. The UK has introduced HPV primary screening ${ }^{11}$, whereby HPV testing for highrisk types is the first test done on a cervical screening sample and only HPV positive samples are then triaged by cytology testing. This increases the detection of cervical intraepithelial neoplasia grade 3 or worse by approximately $40 \%$ compared to liquid-based cytology. Routine screening in the UK takes place every three years (which may change to five years) for women aged 25 to 49 years and every five years for those aged 50 to 64 years.

\section{Conflict of interest}

The author has declared that he has no conflicts of interest. Caldicott Guardian approval (a UK process to ensure that the use of personal data complies with legal requirements for data protection and is in the public interest) was obtained from the Central Manchester University Hospitals NHS Foundation Trust on October 13, 2016, and from North Cumbria University Hospitals NHS Trust on January 17, 2017, Ref CAL/16/015 for data transfer from the Manchester Cytology Centre to the Colposcopy Clinic at the Cumberland infirmary.

\section{Funding}

None

\section{References}

1. Hallam NF, Parker JA. Commentary: HPV Catch-Up Vaccination Reduces the Prevalence of HPV 16 and 18 Infections and Cervical Disease: A Retrospective Study. J Immunological Sci. (2020); 4(3): 1-3

2. Kreimer AR, Herrero R, Sampson JN, et al; Costa Rica HPV Vaccine 
Trial (CVT) Group. Evidence for single-dose protection by the bivalent HPV vaccine-review of the Costa Rica HPV vaccine trial and future research studies. Vaccine. 2018;36 (32, pt A):4774-4782 doi:10.1016/j.vaccine.2017.12.078

3. Sankaranarayanan R, Joshi S, Muwonge R, et al. Can a single dose of human papillomavirus (HPV) vaccine prevent cervical cancer? Early findings from an Indian study. Vaccine. 2018;36(32, Part A):4783-4791. 10.1016/j.vaccine.2018.02.087. - DOI - PubMed

4. Sonawane K, Nyitray AG, Nemutlu GS, et al. Prevalence of Human Papillomavirus Infection by Number of Vaccine Doses Among US Women. JAMA Netw Open. 2019;2(12):e1918571. doi:10.1001/ jamanetworkopen.2019.18571

5. Cameron, R, Kavanagh, K., Pan, J, et al. Human papillomavirus prevalence and herd immunity after introduction of vaccination program, Scotland, 2009-2013. Emerg Infect Dis. 2016; 22(1), 5664. doi:10.3201/eid2201.150736

6. Malagón T, Laurie C, Franco EL. Human papillomavirus vaccination and the role of herd effects in future cancer control planning: a review. Expert Rev Vaccines. 2018;17(5):395-409. doi:10.1080/14 760584.2018.1471986
7. Palmer T, Wallace L, Pollock K, et al. Prevalence of cervical disease at age 20 after immunisation with bivalent HPV vaccine at age 12-13 in Scotland: retrospective population study. 2 doi: 10.1136/bmj.11161 | BMJ 2019;365:11161

8. Drolet M, Bénard E, Perez N, et al. Population-level impact and herd effects following human papillomavirus vaccination programmes: a systematic review and meta-analysis. Lancet 2019. Epub ahead of print: https:// doi.org/10.1016/S0140-6736(19)30298-3.

9. Karube A, Saito F, Nakamura E, et al. Reduction in HPV 16/18 prevalence among young women following HPV vaccine introduction in a highly vaccinated district, Japan, 2008-2017. J Rural Med. 2019; 14: 48-57. - PMC - PubMed)

10. https://www.ema.europa.eu/en/medicines/human/EPAR/ gardasil-9

11. Rebolj M, Janet Rimmer J, Karin Denton K et al. Primary cervical screening with high-risk human papillomavirus testing: observational study. BMJ 2019 Feb 6; 364: 1240. doi:10.1136/bmj. 1240. 